\title{
The role of imaging in the follow up of meningiomas
}

\author{
Timothy J Hodgson, Derek P E Kingsley, Ivan F Moseley
}

\begin{abstract}
A retrospective study of 60 patients with meningiomas was conducted to evaluate the role of imaging in postoperative follow up. Using case notes and imaging studies, requests were assessed with reference to the indications for imaging radiological findings and effect on patient management. Patients were divided into three groups: 34 who had undergone a macroscopically complete resection, 18 with known residual tumour, and eight in whom surgery was not performed. These 60 patients underwent a total of $165 \mathrm{CT}$ and $11 \mathrm{MRI}$ studies. In the complete resection group only two patients developed a recurrence, both having highly suggestive symptoms or signs. It is concluded that routine imaging is not indicated in asymptomatic patients after complete tumour clearance. Both $C T$ and MRI contribute to patient management in those with residual disease, MRI probably being the imaging method of choice.
\end{abstract}

(F Neurol Neurosurg Psychiatry 1995;59:545-547)

Keywords: meningioma; computed tomography; magnetic resonance imaging

Meningiomas are common, benign tumours arising from the meninges lining the brain and spinal cord. They constitute about $20 \%$ of all intracranial tumours, with an incidence of up to seven cases/100 000 population. ${ }^{1}$ As a group, they therefore provide an appreciable demand on neuroimaging resources for initial diagnosis and particularly for follow up. We retrospectively reviewed 60 patients with intracranial meningiomas (52 with a histological diagnosis, eight diagnosed from radiological appearances) to assess the role of radiology in their follow up. The results are analysed and we discuss how imaging studies may be better utilised in following up this substantial group of patients.

Materials and methods

The study consisted of a retrospective review of 60 patients who had had a diagnosis of intracranial meningioma. All had undergone at least one follow up imaging study (CT or MRI) between 1992 and 1994, the cases being selected from the departmental radiological coding system. Of the 12 clinical consultants involved in the group's management, eight were surgeons, four were physicians. Surgery had been performed in 52 patients, whereas the other eight were not considered suitable for surgery and were simply followed up.

The case notes were reviewed and data recorded on a standard form including age, sex, site of original tumour, date of original and subsequent surgery (or diagnosis, if applicable), duration of follow up, and whether the surgeon considered that a macroscopic clearance had been performed and if not, the site of residual tumour. The histology report, including any malignant features, was noted.

The radiology request forms and the relevant CT or MRI images were reviewed. Images taken within 28 days of surgery were not included.

\section{Results}

The study group consisted of 26 men and 34 women, mean age 56 years with a range of $31-80$ and a median of 54 years. The table shows the sites of the meningiomas. The average follow up period was 6.5 years, the range 1 year to 24 years 5 months. Surgery was performed on 52 patients before imaging and there were two deaths.

The 60 patients were divided into three groups: group 1, those who underwent a macroscopic clearance at the initial operation;

\begin{tabular}{|c|c|c|c|}
\hline & \multicolumn{2}{|l|}{ Resection } & \multirow[b]{2}{*}{ Not operated } \\
\hline & Complete & Incomplete & \\
\hline \multicolumn{4}{|l|}{ Supratentorial: } \\
\hline Parasagittal & 10 & 4 & 2 \\
\hline Sphenoid wing & 6 & 3 & 1 \\
\hline Suprasellar & 6 & 2 & - \\
\hline Olfactory groove & 4 & - & - \\
\hline Temporoparietal & 1 & 2 & - \\
\hline Cavernous sinus & - & - & 3 \\
\hline Frontal & 2 & - & - \\
\hline Petrous apex & - & 1 & 1 \\
\hline Optic nerve & - & & - \\
\hline Clivus & - & 1 & - \\
\hline Occipital & - & 1 & - \\
\hline \multicolumn{4}{|l|}{ Infratentorial: } \\
\hline Cerebellopontine & 3 & 3 & 1 \\
\hline Tentorium & 2 & - & - \\
\hline
\end{tabular}


group 2, those known to have residual disease at the end of the operation; group 3, those in whom no surgery was performed due to poor medical condition or tumour inoperability, with follow up only.

Group 1 included 34 patients (56\%) with an average follow up of 6 years 8 months and a range of between 1 year and 24 years 5 months. In total $85 \mathrm{CT}$ studies were performed, the average being 2.5 per patient with a range between one and six. During the review period two MRI studies were performed.

Of the 34 patients, two (6\%) presented with a regrowth of tumour, both having symptoms or signs very suggestive of recurrence. One underwent surgery for an olfactory groove meningioma in 1984 and re-presented in 1988 with heavy nose bleeds. Recurrent meningioma was confirmed on CT. The other underwent surgery for a suprasellar meningioma in 1984, presenting again six years later with a left hemianopia and headaches; MRI and CT confirmed recurrent tumour. Both patients underwent further surgery and to date are well. The original histology of both these cases showed no malignant features. In the remainder of this group the histology disclosed 24 tumours with benign features, and eight had mitoses with malignant findings.

There were another four patients (12\%) who presented with symptoms or signs suggestive of recurrence but in whom no tumour was shown on CT: three had grand mal convulsions one to four years after the initial surgery, the fourth a sixth nerve palsy one year after surgery for a frontal meningioma. In the remaining 28 patients $(82 \%)$ the accompanying request forms stated questionable or no neurology or "routine follow up". Recurrent tumour was not identified on any imaging study. One death unrelated to the meningioma occurred in this group.

Group 2 consisted of 18 patients (30\% of the total) who underwent initially incomplete resection. The average follow up was 5 years 10 months with a range from 1 year 6 months to 14 years 4 months. They underwent a total of $54 \mathrm{CT}$ and nine MRI studies. Of the six patients who had MRI one patient underwent three examinations and another two. There was one death directly related to tumour invasion and sinus occlusion. Patient management was influenced by imaging in seven of the 18 patients, who underwent further surgery, or radiotherapy, or both because of residual or progressive symptoms or signs. Imaging showed unchanged tumour size in nine patients, none of whom had further surgical intervention during the study period. Two patients, one with a suprasellar and the other with a sphenoid wing meningioma, showed some progression in tumour size but neither had further surgery or radiotherapy during the study period. The histology of this group of patients showed 12 that had benign features and six mitoses and malignant features.

Group 3 consisted of eight patients. The average follow up was 7 years 3 months. All follow up imaging was by $\mathrm{CT}$, the average number of studies being 3.3 per patient with a range between one and nine. Only one imaging study resulted in a change of management: four years after initial diagnosis a 75 year old woman with a petrous apex meningioma presented with ataxia, gait disturbance, and headaches; CT showed triventricular hydrocephalus which required shunting. This resulted in rapid clinical improvement.

\section{Discussion}

Meningiomas arise from the meninges and in most cases exhibit benign features. They account for about $14 \%$ to $18 \%$ of all intracranial tumours ${ }^{2}$ with an annual incidence per 100000 population of 2-7 for females and 1-5 for males. ${ }^{1}$ The first reported radiological diagnosis of a meningioma was in 1902, the radiograph requiring a 3.5 minute exposure! ${ }^{3}$ Techniques have since progressed, through ventriculography, pneumoencephalography, and angiography, to the modern day modalities of CT and MRI, which are now the primary method of diagnosis.

The role of imaging in the follow up of meningiomas has not been established. Several publications have reviewed recurrence of meningiomas ${ }^{4-11}$ but only one study used CT or MRI as part of their follow up imaging protocol. ${ }^{5}$ Marks et al reported on 53 consecutive patients after surgery for meningioma. ${ }^{5}$ Four had a recurrence after macroscopic clearance, three with definite clinical evidence, the fourth being asymptomatic. The time interval between initial surgery and recurrence was $1 \cdot 6-3 \cdot 8$ years, contrasting with our study of four to six years. Marks and his colleagues advised yearly CT for the first four years but stated that the use of CT was random, unlike our study in which all patients underwent CT/MRI.

Possible factors in tumour recurrence have been extensively investigated. ${ }^{4-11}$ Sex and age are of no predictive value, ${ }^{4-7}$ whereas tumour site has been claimed to have more importance. Mirimanoff et $a l^{6}$ claim a recurrence rate at five years of $34 \%$ and $30 \%$ for sphenoid ridge and olfactory groove meningiomas respectively whereas other authors state that site is not a useful predictive factor. ${ }^{457}$ Certainly the original site of the tumour is of importance in the ability of the surgeon to perform a macroscopic clearance, with skull base lesions often being impossible to remove completely. In this series, however, few of the skull based tumours, apart from those directly invading the cavernous sinus, could not be completely resected.

The histology of the initial tumour may have a predictive role but again the evidence is confusing. Tumours with syncytial features, mitoses, and areas of focal necrosis are claimed by some groups to show increased recurrence rates ${ }^{510}$ but this has been refuted by others. ${ }^{4}$ In our own study, the two recurrences after complete excision were both originally entirely benign tumours whereas eight 
of the $34(23 \%)$ showed malignant features but did not recur.

One factor which may help to explain recurrence in apparently completely excised tumours is the part played by multicentricity. Borovich and Doron examined radial strips of dura mater removed from the line of attachment of globular meningiomas in 14 consecutive patients. ${ }^{12}$ Meningotheliomatous cells away from the main tumour were demonstrated in all patients, the authors suggesting that there may be a general field change to explain unexpected recurrencies.

The only consistent factor that determines recurrence is initial surgical clearance. In 1957, Simpson devised a grading system based on the degree of excision (grade $1=$ complete excision; grade $5=$ simple decompression). ${ }^{9}$ Mirimanoff et $a l^{6}$ found recurrence free rates at five and 10 years of $93 \%$ and $80 \%$ respectively after a complete resection (Simpson grade 1 and 2) and these figures have been confirmed in other studies. ${ }^{58}$ Our own recurrence free rate after complete tumour clearance was $94 \%$ at six years.

The development of new signs or symptoms should prompt imaging studies (preferably MRI) whether the patient had previously undergone a complete or partial excision. In all our patients who developed new neurology investigation resulted in a change in management. Neither of the two patients in the partially excised group who showed slight tumour progression radiologically had relevant symptomatology and no treatment was undertaken in the study period.

Our conclusions are as follows: (1) patients who have a macroscopic clearance at the initial operation do not require further imaging unless they develop new symptoms or signs. If imaging is required CT or MRI may show recurrence, MRI being preferable due to its multiplanar capability and lack of bone artefact. (2) Patients with known residual disease may be followed up by either CT or MRI, MRI being the imaging method of choice. As in group 1, however, management seems to be related to change in symptoms or signs and not changes on imaging so that little is lost by imaging on clinical indications rather than routinely. (3) Routine imaging is not indicated when a decision has been made not to operate unless the development of new symptoms or signs or new operative techniques would prompt active treatment.

1 Longstreth WT, Dennis LK, McGuire VM, Drangsholt MT, Koepsell TD. Epidemiology of intracranial meningiomas. Cancer 1993;72:639-48.

2 Cushing H, Eisenhardt L. Meningiomas: their classification, regional behaviour, life history and surgical end result. Springfield IL: Charles C Thomas, 1938:785.

3 Mills CK, Pfahler GE. Tumour of the brain localised clinically and by the roentgen rays. Philadelphia Medical fournal 1902;9:268-73.

4 Adegbite AB, Khan MI, Paine KWE, Tan LK. The recurrence of intracranial meningiomas after surgical treatrence of intracranial meningiom

5 Marks SM, Whitwell HL, Lye RH. Recurrence of meningiomas after operation. Surg Neurol 1986;25:436-40.

6 Mirimanoff RO, Dosoretz DE, Linggood RM, Ojemann RG, Martuza RL. Meningioma: analysis of recurrence and progression following neurosurgical resection. $\mathcal{f}$ Neurosurg 1985;62:18-24.

7 Naumann M, Meixenberger J. Factors influencing meningioma recurrence rate. Acta Neurochir (Wein) 1990;107: 108-11.

8 Chan RC, Thompson GB. Morbidity, mortality and quality of life following surgery for intracranial meningiomas. F Neurosurg 1984;60:52-60.

9 Simpson D. The recurrence of intracranial meningiomas after surgical treatment. $₹$ Neurol Neurosurg Psychiatry after surgical treat

10 Crompton MR, Gautier-Smith PC. The prediction of recurrence in meningiomas. $\mathfrak{f}$ Neurol Neurosurg Psychiatry 1970;33:80-7.

11 Jaaskelainen J. Seemingly complete removal of histologically benign intracranial meningioma: late recurrence and factors predicting recurrence in 657 patients. A multivariate analysis. Surg Neurol 1986;26:461-9.

12 Borovich B, Doron Y. Recurrence of intracranial meningiomas: the role played by regional multicentricity. $f$ Neurosurg 1986;64:58-63. 